\title{
Effects of prolonged low-frequency whole-body vibration on rats*
}

\author{
EDWARD L. WIKE and SHARRON S. WIKE \\ University of Kansas, Lawrence, Kans. 66044 \\ and \\ JOSEPH E. WAGNER \\ University of Missouri, Columbia, Mo. 65201
}

Two cages of rats $(N=10)$ were subjected to continuous low-frequency vibration for 92 days. The vibrated Ss weighed less than control $S$ s in the later stages of vibration and were more active in an open-field test given 3 days after the vibration period. Necropsies of both groups did not reveal any permanent damage to the heart, kidneys, spleen, lungs, brain, testes, adrenals, thymus, and liver as a consequence of the prolonged vibration.

Him a $\mathrm{n}$ S $\mathrm{s}$ exposed to low-frequency whole-body vibration in buildings, vehicles, and aircraft often report discomfort. The experimental research regarding the effects of vibration upon animals has been reviewed by Schaefer \& Ulmer (1959) and Nadel (1963). Schaefer, Link, Farrar, \& Weins (1959) showed that lethality in confined rats was directly related to the frequency $(10$ to $45 \mathrm{~Hz})$ of whole-body vibration when the amplitude was held constant at $0.25 \mathrm{in}$. In further studies, Schaefer, Ulmer, \& Link (1959) found that vibrated rats weighted less, were less active in an open field, and that some Ss had testicular atrophy and myocardial hemorrhages. The authors internreted these findings as “... demonstrating a general decrement or debility in both behavioral and physiological functioning, similar to the usual stress pattern [p. 25]."

Hunt \& Quay (1961) and Soskin (1963) employed whole-body vibration to test Hebb's discrepancy-arousal theory of motivation. While their results generally did not confirm Hebb's theory, vibration was shown to be an effective aversive stimulus-Hunt and Quay demonstrated that naive rats would leverpress to terminate vibration, and Soskin observed that rats preferred a nonvibrating goalbox to a vibrating one in U-maze tests. It may be noted that the Ss were vibrated for 53 and 21 days, respectively, in the two studies prior to tests.

More recently, in a series of seven experiments, Wike \& Wike (1972) investigated barpressing that

*This research was supported by a NASA interdisciplinary grant to the University of Kansas, Kenneth Lenzen, principal investigator. We are indebted to Dr. Lenzen, Ensigns Ronald Curry and Eugene Kendall, and Mr. James Stell for material and technical assistance. terminated vibration as a function of vibration frequency $(8$ to $22 \mathrm{~Hz}$ ) and amplitude (from just perceptible to the touch to 0.24 in., peak-to-peak). Escape conditioning was found to be independent of the vibration frequency but directly related to amplitude. Control studies disclosed that escape conditioning could not be accounted for in terms of either the noise or activation produced by vibration. Taken together, the recent research suggests strongly that vibration of sufficient amplitude is an aversive stimulus whose termination is reinforcing.

The present study was a systematic replication of the Schaefer, Ulmer, \& Link (1959) study. In contrast to Schaefer et al, who vibrated confined rats for short periods of time, the ma purpose of the present study was to investigate the behavioral and physiological effects of prolonged low-frequency whole-body vibration upon freely moving rats.

More precisely, two cages of Ss were exposed to vibration continuously for 92 days. During vibration, the Ss' weights and water intakes were monitored. Following the vibration period, activity was measured in an open field, and, thereafter, the Ss were sacrificed and subjected to gross necropsy and microscopic examinations of selected organs for possible permanent damage. Control Ss received identical treatments but were never exposed to vibration.

\section{SUBJECTS}

The Ss were 20 naive Holtzman albino rats, 56 days of age. After receipt from the supplier, the Ss were acclimatized for 12 days prior to vibration with free access to Purina Lab Chow and water. The Ss then were assigned randomly and equally to a vibration and a control group and were housed 5 per cage.

\section{APPARATUS}

The vibration apparatus was an All-American shaker table, Model
100 VA (AH-American Tool \& Manufacturing Co., Chicago, Ill.). Two stainless steel cage pans (16 x $12 \times$ 2.5 in.) were bolted to an 0.75 -in.-thick plywood panel that was attached to the shaker platform. The 12-in.-high cage tops were formed from hardware cloth. The shaker table produced a vibration frequency of $11 \mathrm{~Hz}$ and an amplitude of $0.12 \mathrm{in}$., peak-to-peak. Two identical cages, which housed the control Ss, were placed on a shelf at the same height from the floor as the vibration cages and 18 in. from them. The shaker table, which was mounted on a concrete block support, ran quietly and produced no detectable vibration of the concrete floor or adjoining shelf. The shaker table was housed in a $9 \times 12 \times 10 \mathrm{ft}$ room whose temperature was maintained at $\sim 72^{\circ} \mathrm{F}$ by a window air conditioner.

The open field (45 × $45 \times 20$ in.) was made of 0.62 -in.-thick plywood and had $9 \times 9$ in. squares marked off by black lines.

\section{PROCEDURE}

When the Ss were 68 days of age, vibration was begun and it continued for 92 days. During each of the first 4 days, the shaker was turned off for $20 \mathrm{~min}$ each afternoon to weigh the Ss. Thereafter, the Ss were weighed every third day. At the time of weighing, the cages wer cleaned-every 6 days early in $t$ ! study and every 3 days later on. Each day, the food was replenished and the water bottles were refilled. After Day 40 , the amount of water consumed per cage was measured daily vrior to feeding, etc.

Following 92 days of vibration, the $\sim$ were kept in their cages for 3 days without vibration. On the third day, each $\mathrm{S}$ was given a 3-min open-field test in another experimental room. The number of squares entered by the $S$ was the activity measure. Entry was defined by the S's having all four feet within a square. Two Es simultaneously counted out loud the squares that were entered, and one E tallied the entries. At the time of the test, the Es knew the group membership of only one $S$, and the order of testing the $\mathrm{Ss}$ from the groups was haphazard.

On the following morning, the Ss again were assigned randomly and equally to four cages and transported by an air-conditioned car to the Animal Research Facility, Veterans Administration Hospital, Kansas City, Missouri. That afternoon, the Ss were transported by an air-conditioned van to the Research Animal Diagnostic Laboratory of the University of Missouri at Columbia. The Ss were euthanatized by chloroform overdose and necropsied the evening of the 
same day. The heart, kidneys, spleen, lungs, brain, testes, adrenals, thymus, and liver were removed. Excess adipose tissue; vessels, and extraneous tissue were carefully trimmed from all organs prior to weighing. Paired organs were separately identified: After weighing, the organs were fixed in $10 \%$ buffered neutral Formalin for $24 \mathrm{~h}$, embedded in paraffin, and sectioned at 6 microns. "Tissue sections were routinely stained by hematoxylin and eosin. It should be noted that the veterinary pathologist (J.E.W.) was not informed as to the treatments to which the Ss had been exposed until after his report on the organ weights and microscopic examinations had beén submitted.

\section{RESULTS}

An analysis of variance of the mean body weights for the Ss for blocks of four weighings revealed three significant sources of variation: groups $(\mathrm{F}=5.66, \mathrm{df}=1 / 18, \mathrm{p}<.05)$, blocks $(\mathrm{F}=1461.50, \mathrm{df}=7 / 126, \mathrm{p}<.001)$, and Groups by Blocks $(\mathrm{F}=3.73$, df $=$ $7 / 126, \quad p<.005)$. Simple effects analyses of the interaction disclosed that the groups were clearly separated on the last three blocks of vibration $(\mathrm{Fs}=7.65,10.19,11.26, \mathrm{df}=1 / 144$, ps $<.01, .005, .001$, respectively) with the vibrated Ss being lighter.

The median water intakes per cage for 4-day periods were subjected to an analysis of variance. The lower intake of the Ss in the vibrated cages was at the borderline of significance $(\mathrm{F}=$ $9.14, \mathrm{df}=1 / 2, \mathrm{p}<.10)$, and the periods differed significantly $(\mathrm{F}=$ 4.25 , df $=8 / 16, \mathrm{p}<.01)$. In the case of the comparison between groups, it should be noted that there was only 2 df available for an error estimate. Accordingly, the difference in water intake, despite its marginal level of significance, should not be disregarded.

The final behavioral measure was that of activity in the open field. The number of squares entered was significantly larger $(\mathrm{t}=3.27, \mathrm{df}=18$, $\mathrm{p}<.01)$ for the vibrated $\mathrm{Ss}(\mathrm{M}=80.7)$ than for the control Ss $(M=59.2)$.

As indicated in the procedure section, the heart, kidneys, spleen, lungs, brain, testes, adrenals, thymus, and liver were excised. These organs were weighed, and the organs for the two groups were contrasted with a series of Wilcoxon $\mathrm{T}$ tests. Only one comparison approached statistical significance: the lungs of the vibrated Ss were lighter $(p<.10)$ than those of the control Ss. However, since the rwo groups still differed significantly $(p<.02)$ in body weight at the time of necropsy, the relative weights of all organs were calculated and the groups were compared again. Three comparisons, the left and right kidneys and spleen, were close to significance $(\mathrm{Ts}=84,83,83.5$, respectively; $\mathrm{T}(.10)$ $=83$ ). In each instance, the vibrated Ss had smaller relative organ weights. However, in view of the large number of significance tests that were performed and the marginal levels of significance that were obtained; the organ weight results must be regarded with great caution. Since vibration is of ten regarded as a stressor, there was particular interest in the adrenal weights. Both the right and left adrenals of the vibrated Ss were heavier but not reliably so. Unfortunately, in the case of the adrenals, the sample size was reduced about $20 \%$ because parts of the organs were excised during removal.

The pathologist's reports on the gross and microscopic examinations were collated as a function of organs and experimental treatments. Common microscopic observations included: adrenals-thickening of the zona reticulata; testicles-interstitial endema; liver-binucleism and polypoidy; kidneys-interstitial nephritis and insipissated proteinaceous hyalinized eosinophilic material in some tubules; heart-congestion of the myocardium; and spleen-congestion of the red pulp, extra medullary erythopoiesis, lymphoid hyperplasia, and hemosiderosis. Microscopically, the stomachs were essentially normal but, as would be expected of this species, most Ss evidenced chronic murine pneumonia. However, inspection of the protocols by the Es disclosed no differences between the groups in either the types or frequencies of lesions. Another experienced E likewise examined the microscopic observations and was unable to discern any consistent difference between the groups. ${ }^{1}$

Although all Es concurred in their evaluations of the lesion data, an effort was made to quantify the observations and evaluate them statistically. The microscopic observations were tallied across organs and summed for each S. A Wilcoxon T test of these scores failed to disclose even a trend toward a significant difference $[\mathrm{T}=97 ; \mathrm{T}(.10)=83]$ between the $\mathrm{Ss}$ in the vibrated $(\mathrm{M}=$ $12.3)$ and control $(M=11.0)$ groups. Furthermore, inspection of the frequencies on an organ-by-organ basis revealed that additional tests at the individual organ level would be pointless.

\section{DISCUSSION}

In accord with the studies by Schaefer, Ulmer, \& Link (1959), we found that vibrated $S$ s weighed less than control Ss. These authors also reported that lowered intake of food was associated with vibration. In the present study, a difference, at the marginal level of significance, was observed in water intake, with the vibrated Ss drinking less. Taken together, these results suggest that vibration is an aversive stimulus that disturbs consummatory behavior.

The findings of the two investigations were clearly in disagreement in two instances-activity and physiological damage. In the present study, the vibrated Ss were more active than the control Ss in an open-field test, while the vibrated Ss of Schaefer et al were less active. Since the studies differed in many procedural details, it is somewhat speculative as to what might account for these conflicting results. Two significant procedural details should be mentioned, however. First, Schaefer et al permitted less time ( 1 day vs 3 days) for their Ss to recover from vibration before testing them in an open field. They also noted some disturbances in locomotion and unusual backing-up movements. Our $\mathrm{Ss}$, on the other hand, with a longer recovery period, did not manifest such behaviors. Second, Schaefer et al imposed a vibration that was twice the amplitude of ours. Moreover, their Ss were firmly confined to small individual compartments, while our Ss were unconfined in cages. Thus, the Ss in the present study could partially dampen out the effects of the vibration by moving about and huddling together. On the basis of earlier investigations (Wike \& Wike, 1972), we would suspect that the differences in these two studies in vibration amplitude and the mode of its presentation were the important factors underlying the conflicting activity findings. How can increased activity following vibration be accounted for? Perhaps the long-term vibration served to diminish normal movement in the living cages and thereby created a greater "need" for activity. Although this interpretation is a post hoc one, it is testable. If groups of Ss were vibrated for different durations, then activity in an open field should vary directly with the length of the vibration period.

Schaefer et al found that some of their vibrated Ss suffered testicular atrophy and myocardial hemorrhages. The microspic examinations of our Ss revealed instances of interstitial endema in the testes and congestion of the myocardial vessels, but these conditions were not observed more frequently in the vibrated Ss. In fact, the vibrated and control Ss did not appear to differ on any of the organs that were examined. Once again, we would suspect that imposition of a lower amplitude of vibration upon unconfined Ss was less stressful than 
the vibration used by Schaefer et al. The major finding of the present investigation was that $\mathrm{Ss}$ could be vibrated continuously for over 3 months without any apparent significant physiological effects.

In view of the failure to find physiological differences between the two groups, the question might be raised as to whether or not the vibration amplitude was sufficient for it to serve as an effective stimulus. Since the vibrated $S$ s weighed less, appeared to drink less, and were more active in an open field, there is evidence within the study to suggest that the vibration was an effective stimulus. Furthermore, Soskin (1963) found that rats avoided a vibrating goalbox with an amplitude of 369.5 microns and Wike \& Wike (1972, Experiment 4) observed that Ss with an intermediate amplitude of vibration (0.12 in.) made significantly more barpresses to terminate vibration than did a low amplitude group (just perceptible to touch). Thus, there is evidence, both internal and collateral, to support the contention that the vibration used in the present investigation was an effective stimulus.

\section{REFERENCES}

HUNT, J. M., \& QUAY, H, C. Early vibratory experience and the question of innate reinforcement value of vibration and other stimuli: A limitation on the discrepancy (burnt soup) principle in motivation. Psychological Review, 1961, $68,149-156$.

NADEL, A. B. Vibration. In N. E. Burns, R. M. Chambers, and E. Hendler (Eds.) Unusual environments and human behavior. New York: Collier-Macmillan, 1963. Pp. 379-394.

SCHAEFER, V. H. LINK, H. J, FARRAR. J. U., \& WEINS, D. Lethality in rats as a function of frequency in constant-displacement vibration. USA Medical Research Laboratory Report No. $390,1959$.

SCHAEFER, V. H., \& UIMER, R. G. A representative bibliography of research in low-frequency mechanical vibration. USA Medical Research Laboratory Report No. $405,1959$.

SCHAEFER, V. H., ULMER, R. G., \& LINK, H. J. Some behavioral and physiological studies in vibration. USA Medical Research Laboratory Report No. 389,1959 .

SOSKIN, R. A. The effects of early experience upon the formation of environmental preferences in rats. Journal of Comparative \& Physiological Psychology, 1963, 56, 303-306.

WIKE, F. L., \& WIKE, S. S. Escape conditioning and low-frequency whole-body vibration: The effects of frequency, amplitude, and controls for noise and activation. Psychonomic Science, 1972, 27, 161-164.

NOTE

1. We wish to thank Dr. Don R. Justesen, Neuropsychology Laboratory, V.A. Hospital, Kansas City, Mo., for examining these data. 